\title{
Myasthenia gravis in a professional cyclist-A case report
}

\author{
Hiroteru Hayashi", Hiroki Funasaki, Kentaro Kawai, Sakiko Ito, Keishi Marumo
}

Department of Sports and Wellness Clinic, Jikei University School of Medicine, Tokyo, Japan;

*Corresponding Author: hiroteru17@gmail.com

Received 2 October 2013; revised 2 November 2013; accepted 10 November 2013

Copyright (C) 2013 Hiroteru Hayashi et al. This is an open access article distributed under the Creative Commons Attribution License, which permits unrestricted use, distribution, and reproduction in any medium, provided the original work is properly cited.

\begin{abstract}
Since myasthenia gravis affects the muscular system, athletes suffering from the disease often have difficulty in performing or returning to their sports activity, in which exertion of continuous muscular power is required. In this paper, we present a case of a professional bicycle rider with symptomatic MG who successfully return to his sport activity after thymectomy, steroid pulse therapy and aggressive rehabilitation. He resumed load training, returned to competitive cycling 8 months after leaving hospital while being on $20 \mathrm{mg}$ PSL/day, and won seven victories in the top class cycling competitions. This is the first report on a professional athlete suffering from MG who successfully returned to competitive sports after aggressive steroid treatment.
\end{abstract}

Keywords: Myasthenia Gravis; Aggressive Rehabilitation; Return to Sports

\section{INTRODUCTION}

One of characteristic symptoms of myasthenia gravis (MG), an autoimmune disease of the neuromuscular junction is the daily variation in fatigability. Since myasthenia gravis affects the muscular system, athletes suffering from the disease often have difficulty in performing or returning to their sports activity, in which exertion of continuous muscular power is required [1]. We present a case of a professional bicycle rider with symptomatic MG who successfully return to his sport activity after thymectomy, steroid pulse therapy and aggressive rehabilitation. The available literature on treatment outcomes in athletes suffering from MG is reviewed and discussed.

\section{CASE REPORT}

A 22-year-old professional bicycle rider presented to the thoracic surgery department at our hospital with a mediastinal tumor detected on a routine medical examination. His chest X-ray, CT and MRI (Figure 1) indicated thymoma and blood analysis showed increased anti-acetylcholine receptor (anti-AchR) antibody levels $(4.1 \mathrm{nmol} / \mathrm{l})$. A diagnosis of myasthenia gravis was made and after removal of the tumor by video-assisted thoracic surgery the patient was discharged from the hospital.

He had not shown any symptoms affecting his daily life activities for few weeks after the discharge, but difficulty in head elevation and looking straight ahead developed at seven weeks following surgery. He was rehospitalized due to the MG remission manifested by progressive muscle weakness of his neck and tights, waning phenomenon and increased anti-AchR antibody levels (up to $43.0 \mathrm{nmol} / \mathrm{l}$ ). The grade of MG at re-admission was IIA type according to the Osserman classification [2] and class IIA by the MGFA classification [3].

\section{COURSE AFTER RE-ADMISSION (FIGURE 2)}

Prednisolone (PSL) treatment was initiated and the dose was gradually increased from $15 \mathrm{mg} /$ day to $80 \mathrm{mg} /$

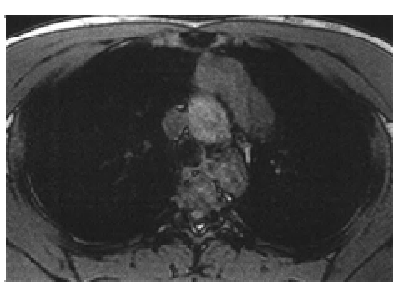

(a)

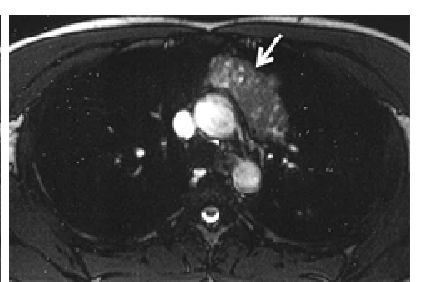

(b)
Figure 1. Chest MRI (transverse image) before operation. (a) T1WI; (b) T2WI. In T2WI image, a solid tumor with a low signal intensity containing a nodular liquid inclusion can be found in the anterior mediastinum (white arrow). 


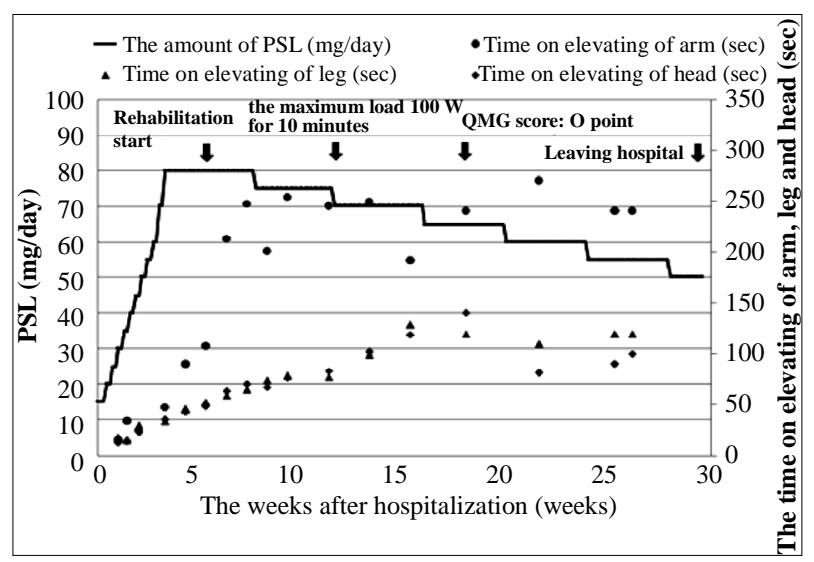

Figure 2. Clinical course after re-hospitalization. PSL was gradually increased from $15 \mathrm{mg} /$ day up to $80 \mathrm{mg} /$ day maximum during one month. Then, steroid pulse therapy was started and followed by weaning from the steroid by decreasing PSL dose by $5 \mathrm{mg}$ /day every fourth week. Rehabilitation was started from six weeks after re-hospitalization.

day maximum; the PSL administration was continued for one month. Steroid pulse therapy $[4,5]$ was then started and was followed by weaning from the steroid done by decreasing PSL dose by $5 \mathrm{mg} /$ day every fourth week. The patient strongly wished to return to competitive cycling. Although still on $80 \mathrm{mg} /$ day PSL, his generally good state allowed rehabilitation. It was initiated early in the course of hospitalization: the initial $10 \mathrm{~W}$ load (measured with an ergometer) was applied for 5 min and then gradually increased every $3^{\text {rd }}$ day to reach the maximum of $100 \mathrm{~W}$ for $10 \mathrm{~min}$. The exercise frequency was set at 5 times/week, and each time muscular power, respiratory rate and pulse were monitored. During rehabilitation exercises the patient was asked to wear a mask to prevent infection. Later, he developed a "moon face", steroidinduced acne and a slight rise in ocular tension in both eyes (all lasted temporarily) but no other serious complications (severe infection, crisis, etc.) were observed. Although the QMG score [3] was 11 points at the time of admission, it improved to zero at 17 weeks.

\section{COURSE AFTER DISCHARGE}

Following discharge, the patient continued to take 50 $\mathrm{mg}$ /day PSL for total 29 weeks from the beginning of the steroid therapy. He resumed load training, returned to competitive cycling 8 months after leaving hospital while being on $20 \mathrm{mg}$ PSL/day, and won seven victories in the top class cycling competitions. The weaning from initial steroid dose has continued for 3 years and 5 months and now the PSL dose has been reduced to $6 \mathrm{mg} / \mathrm{ml}$.

\section{MUSCLE STRENGTH, QUANTITY AND CHANGES IN THE BONE DENSITY}

In order to evaluate muscle strength, we measured isokinetic strength of muscles in knee flexion and extension by Cybex (CBX-770, Cybex Co.) and cross-sectional areas of the trunk and thigh muscles were assessed for muscle quantity using abdomen and thigh CT scans. Furthermore, bone density by DEXA method was measured and evaluated for time-related changes.

Although slight variations in muscle strength per weight at knee extension were found, there were almost no changes observed between the values collected at 1 and 3 months following the discharge. The muscle strength at knee extension increased even to $109 \%$ of the one-month follow-up value at the time of return to full sports activity. On the other hand, the muscle strength at knee flexion decreased to $86 \%$ of the one-month follow-up value at 3 months following the discharge. Then, it gradually increased in parallel to the decreasing dose of the steroid and reached an almost equivalent value as measured at one month following discharge at the time of return to sports (Figure 3).

The femoral CT axial scans taken at the time of return to sports (the upper patellar border level) demonstrated an increase in the tissue mass of the vastus medialis, biceps femoris, semitendinosus, semimembranosus and the sartorial muscles when the muscle cross sections were compared with those at the time of discharge. The amount of the tissue mass in the femoral CT axial scans was the average value obtained from 3 measurements by the PACS method and freehand ROI selections.

The abdominal CT axial scans (at the level of inferior margin of the $4^{\text {th }}$ lumbar vertebra) showed a remarkable

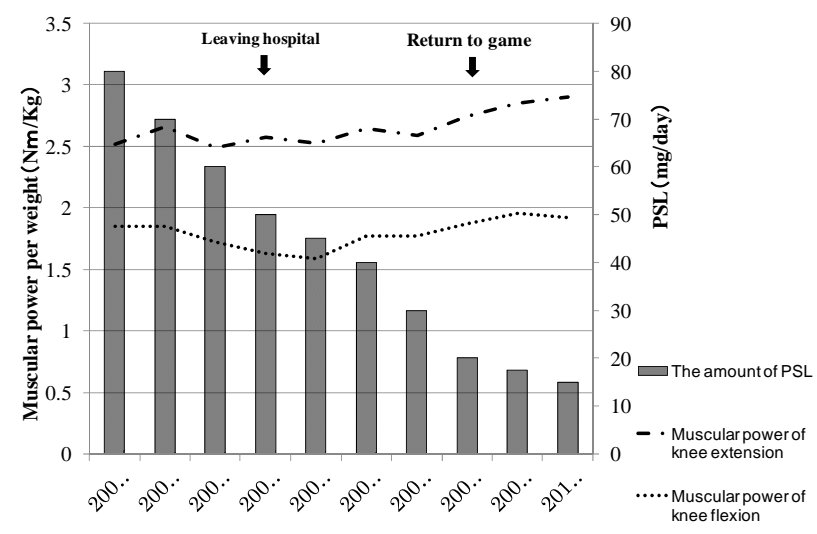

Figure 3. PSL dose and its relationship with the knee extension-and-flexion muscular power measured with a muscular power-measuring device (CBX-770 by Cybex). Although slight variations in muscle strength per weight at knee extension were found, there were almost no changes observed between the values collected at 1 and 3 months following the discharge. Then, muscular strength gradually increased. On the other hand, the muscle strength at knee flexion decreased at three months following the discharge. Then, it gradually increased in parallel to the decreasing dose of the steroid and reached an almost equivalent value as measured at one month following discharge at the time of return to sports. 
decrease in visceral fat at 3 years and 3 months after return to full sport activities when compared to the value at the time of first return $(79.6 \mathrm{~cm}$ and $89.1 \mathrm{~cm}$, respectively; PACS measurement method). Moreover, although marked tissue mass increases were not observed in cross sections of the abdominal internal oblique, abdominal external oblique, transverse abdominal and rectus abdominis muscles, morphology of the muscle tissue changed accordingly with the overall decrease in the visceral fat (Figures 4 and 5).

Bone mineral density was maintained within the normal range from the time of re-hospitalization until 9 months following return to competitive cycling; it reached its minimum value of $1.292 \mathrm{~g} / \mathrm{cm}^{2}$ at 2 months after re-hospitalization, when the PSL dose was the highest. Than, it gradually increased reaching YAM (young adult mean) score of $112 \%$ ( $T$ score: 1.8 ) and $105 \%$ by the contemporary comparison method (Z score: 0.7 ) (Figure 6).

\section{DISCUSSION}

Myasthenia gravis is a disease characterized by progressive loss of muscle strength and easy fatigability; prognosis depends on the type of disease [2,6,7]. Definite treatment has not yet been established but administration
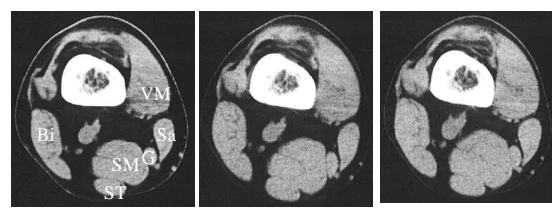

(a) $\mid$ (b) $\mid$ (c)

(d) (e)
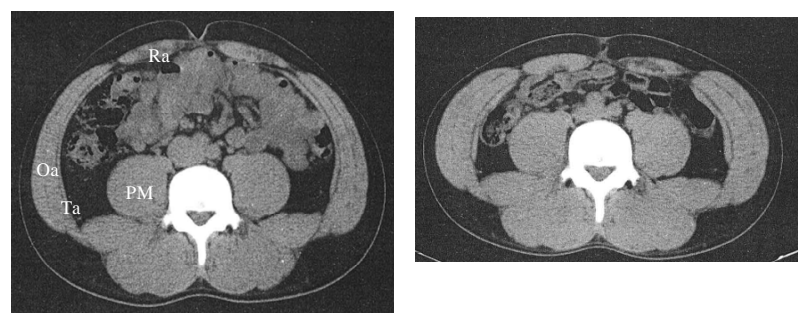

Figure 4. CT (transverse images). ((a), (b), (c)). CT scans of the thigh at the level of superior patellar border (VM: medial vastus muscle, Bi: biceps femoris, ST: semitendinosus, SM: semimembranosus, Sa: sartorius muscles). (a) CT scan taken at the time of discharge (PSL $50 \mathrm{mg} / \mathrm{day}$ ), (b) CT scan taken at the time of return to competitive cycling (PSL $20 \mathrm{mg} / \mathrm{a}$ day), (c) CT scan taken 3 years and 3 months after return to full sports activity (PSL $7 \mathrm{mg} /$ day); ((d), (e)) Abdomen CT scans at the level of inferior-border L4 (Ra: rectus abdominis, Oa: inner and external oblique, Ta: transverse muscle of abdomen, PM: greater psoas muscles); (d) CT scan taken at the time of return to competitive cycling (PSL $20 \mathrm{mg} /$ day), (e) CT scan taken at 3 years and 3 months after return to full sports activity (PSL 7 mg/day). Increase in the muscle cross-sectional area of VM, Bi, ST, SM, and Sa was observed. Moreover, the amount of visceral fat decreased. On the other hand, almost no changes were observed in the muscle cross-sectional area of Ra, Oa and Ta.

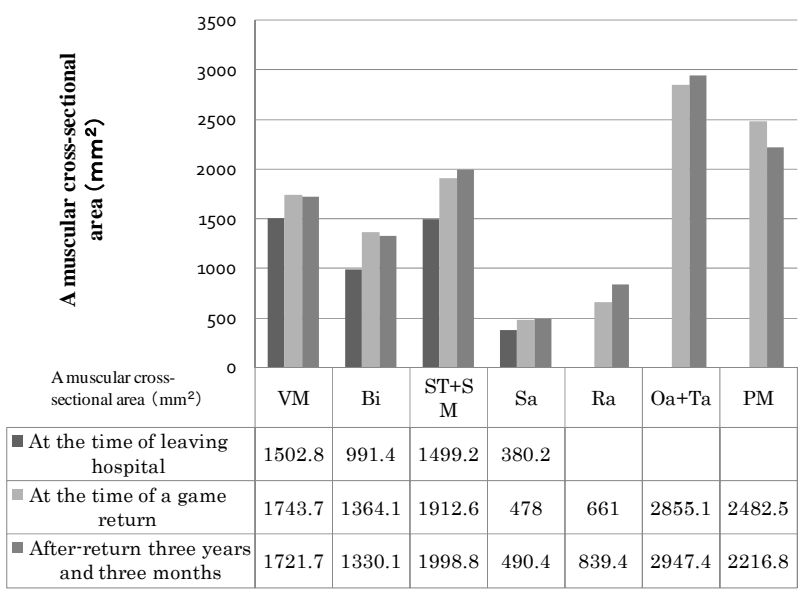

Figure 5. Results of CT measurements of muscle cross-sectional areas $\left(\mathrm{mm}^{2}\right)$. Although each muscle cross-sectional area had gradually increased from the time of discharge to the time of return to full sports activity, it did not show any changes for the following 3 years and 3 months.

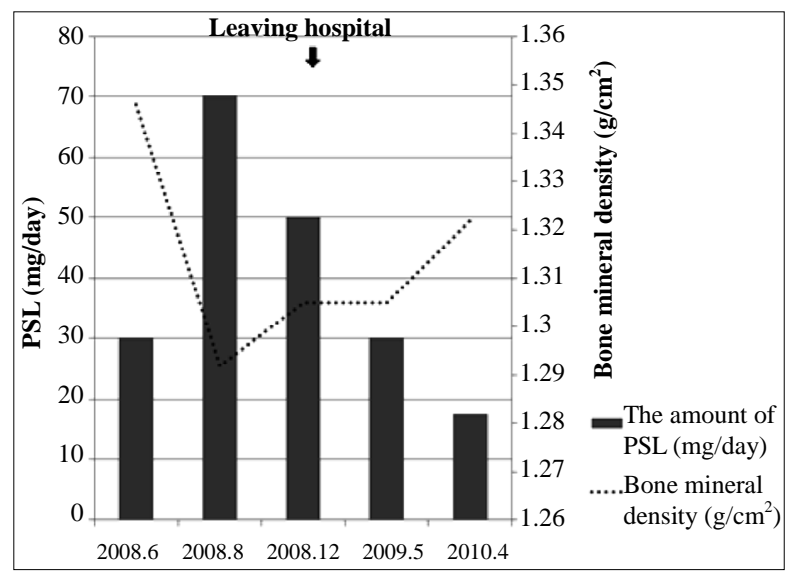

Figure 6. The PSL dose and bone density (the DEXA method). Although bone mineral density reached its minimum value at 2 months after re-hospitalization it could be maintained within the normal range from the time of re-hospitalization until 9 months following return to competitive cycling.

of a cholinesterase inhibitor or medium dose PSL has been shown to induce spontaneous remission in Osserman classification type I and MGFA classification class 0 , I cases [8]. In more advanced stages, thymectomy and long-term PSL therapy are usually applied (cases with Osserman classification type II and MGFA classification class II - V complicated by thymoma); treatment results are greatly influenced by patient compliance and side effects of the applied therapy. The presented case was classified as A type (Osserman) and class IIA (MGFA) and steroid-pulse therapy was applied for 7 months to the patient who had also undergone thymectomy.

Generally, it is difficult for a top athlete suffering from and treated for MG to return to sports activity in disciplines that require muscular power and endurance like 
Table 1. Three reports on return to sports in MG patients.

\begin{tabular}{ccccccc}
\hline Author & Year e publication & Old & Sex & MG classification & Sports & Level \\
\hline Scheer BV et al. & 2012 & 52 & Male & Mild generalized (MGFA IIA) & Ultra marathon & Recreation \\
Leddy JJ et al. & 2000 & 17 & Male & Mild generalized & Football & College \\
Hayashi H et al. & 2013 & 22 & Male & Mild generalized (Osserman IIA) (MGFA IIA) & Bicycle rider & Professional \\
\hline
\end{tabular}

race cycling. This is not only because of temporary discontinuation of training caused by long-term hospitalization but also because of the nature of the disease and its complications (ex. crisis). In many cases, side effects [9-11] such as osteoporosis and loss of muscle strength that might occur due to large-dose steroid therapy may pose additional problems [1].

In fact, only two reports on athletes affected by MG and their return to sports activity have been published to date [12,13] (Table 1). In both cases, patients had mild generalized MG (class IIA, MGFA) as in our case. Though their sports disciplines required less muscle strength and endurance than professional cycling (American football and marathon, respectively) and the level of activity was comparatively lighter than competitive cycling (one was a student practicing at a college sports club and another trained recreationally), in both cases full return to pre-disease sports activity was not possible. This is the first report on successfully treated professional athlete with MG class IIA (MGFA classification) who was able to return to his full pre-disease sports activity following steroid-pulse treatment.

We presume that three factors played important roles in treatment to full recovery in our case. First, patient's compliance was excellent and drawn by the strong desire to return to pre-disease sports activity. Second, rehabilitation was initiated early, when the patient was still under large doses of PSL. Therefore, loss in muscle strength and volume could be minimized and severe complications (e.g. crisis) avoided. Third, due to young age of the patient, his originally high bone mineral density was maintained within the normal range during the course of treatment. This allowed load training during rehabilitation.

\section{CONCLUSION}

We report a case of a professional bicycle rider who achieved full recovery to competitive sports after re-activation of myasthenia gravis (type II Osserman classification and class IIA, MGFA classification). Treatment including steroid-pulse therapy and early rehabilitation program started while the patient was still on high dose steroids and during hospitalization. This is the first report on a professional athlete suffering from MG who successfully returned to competitive sports after aggressive steroid treatment.

\section{REFERENCES}

[1] American Academy of Pediatrics. Committee on Sports Medicine and Fitness (1994) Medical conditions affecting sports participation. Pediatrics, 94, 757-760.

[2] Osserman, K.E., et al. (1971) Studies in myasthenia gravis-Review of a twenty-year experience in over 1200 patients. Mount Sinai Journal of Medicine, 38, 497-537.

[3] Jaretzki $3^{\text {rd }}$, A., et al. (2000) Myashenia gravis: Recommendation for clinical research standards. Task Force of the Medical Scientific Advisory Board of the Myasthenia Gravis Foundation of America. The Annals of Thoracic Surgery, 70, 327-334.

http://dx.doi.org/10.1016/S0003-4975(00)01595-2

[4] Lindberg, C., et al. (1998) Treatment of myasthenia gravis with methylprednisolone pulse: A double blind study. Acta Neurologica Scandinavica, 97, 370-373. http://dx.doi.org/10.1111/j.1600-0404.1998.tb05968.x

[5] Arsura, E., et al. (1985) High-dose intravenous methylprednisolone in myasthenia gravis. Archives of Neurology, 42, 1149-1153.

http://dx.doi.org/10.1001/archneur.1985.04060110031011

[6] Grober, M., et al. (1998) Self-care actions to manage fatigue among myasthenia gravis patients. Journal of Neuroscience Nursing, 30, 191-199.

http://dx.doi.org/10.1097/01376517-199806000-00007

[7] Oosterhuis, H.J. (1981) Observation of the natural history of myasthenia gravis and the effect of thymectomy. Annals of the New York Academy of Sciences, 377, 678-690. http://dx.doi.org/10.1111/j.1749-6632.1981.tb33766.x

[8] Masaoka, A., et al. (1996) Extended thymectomy for myasthenia gravis patients: A 20-year review. The Annals of Thoracic Surgery, 62, 853-859. http://dx.doi.org/10.1016/S0003-4975(96)00376-1

[9] Sghirlanzoni, A., et al. (1984) Myasthenia gravis: Prolonged treatment with steroids. Neurology, 34, 170-174. http://dx.doi.org/10.1212/WNL.34.2.170

[10] Pascuzzi, R.M., et al. (1984) Long-term corticosteroid treatment of myasthenia gravis: Report of 116 patients. Annals of Neurology, 15, 291-298. http://dx.doi.org/10.1002/ana.410150316

[11] Kanda, F., et al. (2001) Steroid myopathy; pathogenesis and effects of growth hormone and insulin-like growth factor-I administration. Hormone Research, 56, 24. http://dx.doi.org/10.1159/000048130

[12] Leddy, J.J., et al. (2000) Myasthenia gravis in a collegiate football player. Medicine \& Science in Sports \& Exercise, 
32, 1975-1979.

http://dx.doi.org/10.1097/00005768-200012000-00001

[13] Scheer, B.V., et al. (2012) Myasthenia gravis and endur- ance exercise. American Journal of Physical Medicine \& Rehabilitation, 91, 725-727.

http://dx.doi.org/10.1097/PHM.0b013e31824663c6 